\title{
The Effects of Metallic Loop-Like Accessory Worn on the Human Body on SAR at $2.45 \mathrm{GHz}$
}

\author{
'Bioelectromagnetics Research Group (BioEM), \\ ${ }^{2}$ Advanced Communication Engineering (ACE), \\ School of Computer and Communication Engineering, Universiti Malaysia Perlis (UniMAP), Kampus Pauh Putra, Perlis, Malaysia \\ ${ }^{3}$ Dept of Electronics Engineering Technology, Faculty of Engineering Technology,Universiti Malaysia Perlis,Malaysia \\ ${ }^{4}$ Centre of Diploma Studies (CDS),Universiti Malaysia Perlis (UniMAP), Campus UniCITI Alam Sg. Chuchuh,Perlis,Malaysia
}

H. H. Zainal ${ }^{1}$, H. A. Rahim ${ }^{\prime *}$, Soh Ping Jack ${ }^{2}$, K.N.Abdul Rani ${ }^{3}$, H. Lago ${ }^{2}$, M.S.Zulkefli ${ }^{3}$, and N.F.N.Yah ${ }^{1}$

\begin{abstract}
This paper presents the Specific Absorption Rates (SAR) in the human body with a monopole antenna.The distance between the antenna and the body were varied at different distances. The parameters $(\varepsilon r, \sigma)$ used in the human body set according to the standard tissue equivalent liquids recommended by the IEEE and FCC. The simulations were made by means of CST Microwave Studio software at frequencies of $2.45 \mathrm{GHz}$. The effect of the body on the SAR calculation in the body were measured. The SAR values were recorded in term of SAR for $10 \mathrm{~g}$ of tissue. The TM is positioned against the metallic loop-like accessory, place on the left wrist of the generic arm at a varied distance from the cylindrical phantom. Numerical analysis conducted using a broadband textile monopole antenna (TM) with variations of orientation and distance showed that SAR values increased when the TM is horizontally polarized.
\end{abstract}

\section{Introduction}

Recently, wearable textile antenna is important in providing comfort to users in wireless body area network(WBAN). The main requirement for wearable textile antennas is the use of flexible materials for ease integration into clothing.

A wearable textile antenna requires such as it must be comfortable, lightweight and inexpensive for their common use to become actuality. Because the wearable antenna will be placed in the closeness of human body, it is necessary to consider the interaction between the human body and antenna.

In additional, when the antenna is mounted on the body, the reflection coefficient, radiation pattern and efficiencies of antenna are afflicted due to the lossy nature of human skin. Therefore, the antenna's performance will be degraded when it is located on the body compared to its distance away from body.

To protect the human being body from radio wave exposure, antennas used in WBAN's must have low SAR based on the standard SAR limit which is $2 \mathrm{~W} / \mathrm{kg}$ averaged over $10 \mathrm{~g}$ of tissue following IEC standard for wireless device. Thus, this project is performed in order to evaluate the realistic position and distances of metallic loop like accessory worn on the body against the wearable textile antenna.

Specific absorption rate is a measure of heat syndication in a body medium by an antenna. It describes the absorption of the electromagnetic energy by human tissues and is defined as follows ${ }^{1}$ :

$$
\mathrm{SAR}=\frac{\sigma|E|^{2}}{\rho} \quad(W / k g)
$$

where $E$ is the electric field induced in the tissue, $\mathrm{V} / \mathrm{m}, \sigma$ is the conductivity of the tissue, $\mathrm{S} / \mathrm{m}$ and $\rho$ is the tissue density, $\mathrm{kg} / \mathrm{m} 3$.
Most of previous research has been focusing on the effect of metallic objects. Metallic objects such as spectacles, earing and ring is no longer a symbol of wealth, but it is a necessity to every individual. A dental implant positioned near the source had higher effect on local SAR, which has also been observed in previous study $^{2}$. The authors reported that the metal implant positioned in second premolar in the mandible and the first molar in the maxillary at $900 \mathrm{MHz}$ affected the current density more than other position.

$\mathrm{In}^{3}$, it concludes that different value of dielectric properties inside the body give influence on the performance of antenna. The performance of antenna (return loss and radiation pattern) is significantly affected by the presence of the body.

In previous research, the authors have found that part of energy radiated by the mobile phone antenna is absorbed by the human body, head and hand ${ }^{4}$. SAR of mobile phone consumed by humans is very influenced by the mobile network carrier, characteristics of mobile phone and antenna, antenna positioning, and the extended power from the mobile phone ${ }^{5}$.

$\operatorname{In}^{6}$,they described that a metallic object can increase or decrease SAR values. Obviously, the maximum SAR can be increased up to 6 times comparing with no object condition by a ring shape object, 4.7 times by a triangle and 5 times by a square object.

Therefore, to investigate the interaction between the wearable device, i.e., antenna and the human body, a cylindrical phantom model is used in the simulation to human body structure. Numerical simulations are conducted using CST Microwave Studio based on the Finite Integral Technique (FIT). The generic human body consists of three cylindrical bricks, representing the upper human body torso and two arms.

* Corresponding author: haslizarahim@unimap.edu.my 


\section{Method}

In this paper, textile monopole antenna is employed as the radiating source, which operates at $2.45 \mathrm{GHz}$. The antenna consists of $0.17 \mathrm{~mm}$ thickness of metallic layer (copper) with dielectric constant $E r=1.43$,loss tangent of 0.025 and thickness of $1.7 \mathrm{~mm}^{7}$. A detail description on the antenna design can be found $\mathrm{in}^{7}$. The SAR values resulting from the radiating textile monopole antenna are benchmarked against two scenarios, which are with and without presence of metallic loop-like accessory1Watts input power was applied to the textile monopole antenna for both frequencies.
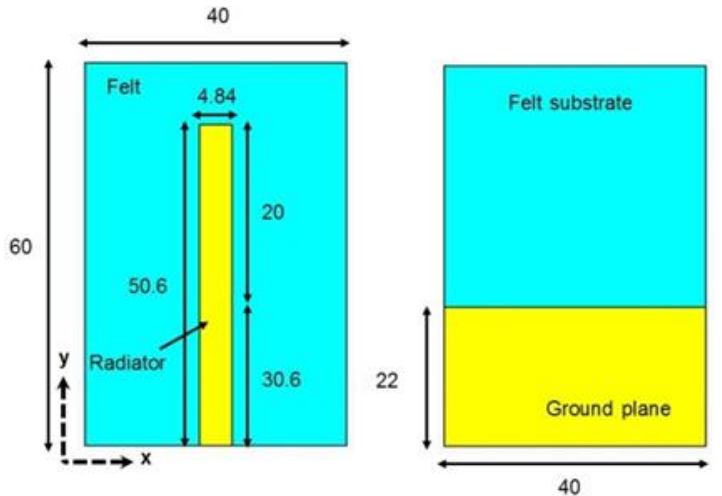

Figure 1 : TM antenna ${ }^{7}$

\subsection{Human Torso Model}

In this paper, the human body is modeled as cylindrical phantom made available by CST Microwave Studio. The frequency dependent parameter $\left(\varepsilon_{r}, \sigma\right)$ use in human body tissue were the same as recommended by IEEE and $\mathrm{FCC}^{8}$, as listed in Table 1

Table 1.Dielectric Properties of Body Tissue at 2.45

\begin{tabular}{|c|c|c|}
\hline $\begin{array}{c}\text { Frequencies } \\
, \mathrm{GHz}\end{array}$ & $\varepsilon_{r}$ & $\sigma(\mathrm{S} / \mathrm{m})$ \\
\hline 2.45 & 53.57 & 1.81 \\
\hline
\end{tabular}

\subsection{The Metallic Loop like Accessory Items}

In order to study the impact of the metallic items on the human body tissues, metallic loop like accessory were modeled as conducting objects (silver) for both frequencies respectively. However in this study, the edge of metallic loop like accessory was closely attached to the cylindrical model (Figure 2).

The distance between the human arm and textile antenna was varied to $4,8,12,16$ and 20 while the metallic loop like accessory was fixed at the same position in each case investigated. TM antenna was oriented in two different direction, i.e. ,vertical and horizontal .

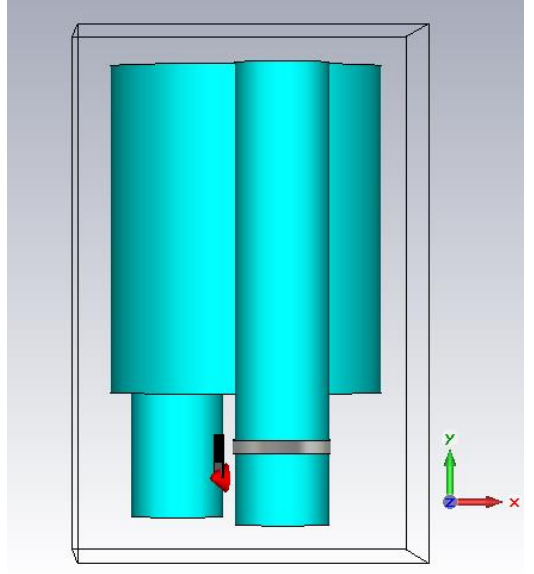

Figure 2: TM in position horizontally

\section{Result}

Figure 3 shows the peak SAR values inside the human body with and without the presence of metallic loop like accessory at frequency $2.45 \mathrm{GHz}$ in term $10 \mathrm{~g}$ SAR. The highest SAR value when the antenna horizontally orientation at distances $4 \mathrm{~mm}$ which is $23.69 \mathrm{~W} / \mathrm{Kg}$. The max SAR value decrease $90 \%$ when the antenna vertically orientation. That can be conclude, the amount of energy absorbed inside the human body is noticeably decreased when the distance between the metallic loop like accessory and the human body is increased.

Figure 3 clearly showed that a metallic loop like accessory that was worn in the close proximity to the radiating source noticeably affects the SAR distribution. The maximum SAR generally appears in the area where the metallic loop like accessory is in the closest distances from the body and occurs when antenna is horizontally polarized.

For example, the value of max $10 \mathrm{~g}$ SAR (4.79 $\mathrm{W} / \mathrm{Kg}$ ) occurs when antenna is horizontally polarized and mounted $16 \mathrm{~mm}$ from the metallic item but when the antenna vertically polarized and placed $16 \mathrm{~mm}$ from metallic loop like accessory the value of SAR is small which is $0.65 \mathrm{~W} / \mathrm{Kg}$ only.It shows the antenna orientation could decreased the SAR value by approximately 7 times compared to horizontally case.



Figure 3 : $10 \mathrm{~g}$ SAR 
Figure 4 show the variations on antenna resonant frequency due to the presence of metallic loop like accessory at frequency $2.45 \mathrm{GHz}$. It can be clearly seen that the presence of metallic loop like accessory has detuned the resonant frequency up to $3 \%$ at $2.45 \mathrm{GHz}$ .However, the detuning effect may vary depending on the distance between the antenna and metallic loop like accessory. The worst case of $\mathrm{S}_{11}$ is observed when TM is horizontal polarization, which is parallel to the human body model with the distance to the metallic item, i.e, 8 $\mathrm{mm}$ and $12 \mathrm{~mm}$.

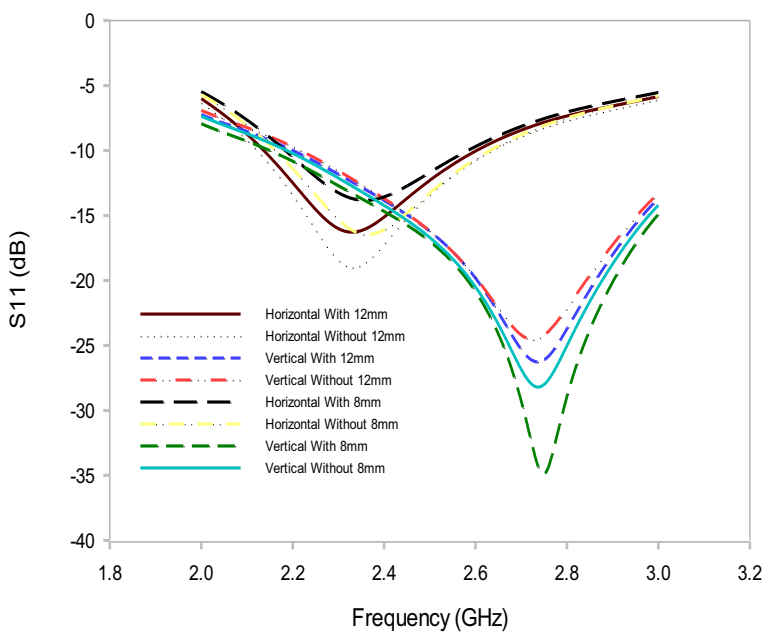

Figure $4: S_{11}$ at $2.45 \mathrm{GHz}$

Figure 6 show the changes of the gain for two situation, i.e.,1) with metallic loop like accessory,2) without metallic loop like accessory with two orientation such as horizontally and vertically. The gain value increase for entire vertically polarized antenna case investigated.

In addition, the results have shown that the antenna gain in frequency $2.45 \mathrm{GHz}$ is significantly improved as the separation distance between the antenna and human body, distance increase in all position.

From the plot, the maximum gain of more than $6 \mathrm{dBi}$ was obtained between 4 and $20 \mathrm{~mm}$. From the Figure 5, the results show that the gain of the initial before attached with metallic loop like accessory with horizontally polarized was $3.9 \mathrm{dBi}$. However, in the presence of metallic loop like accessory ,the gain of the antenna gain was increased by 1.5 times.

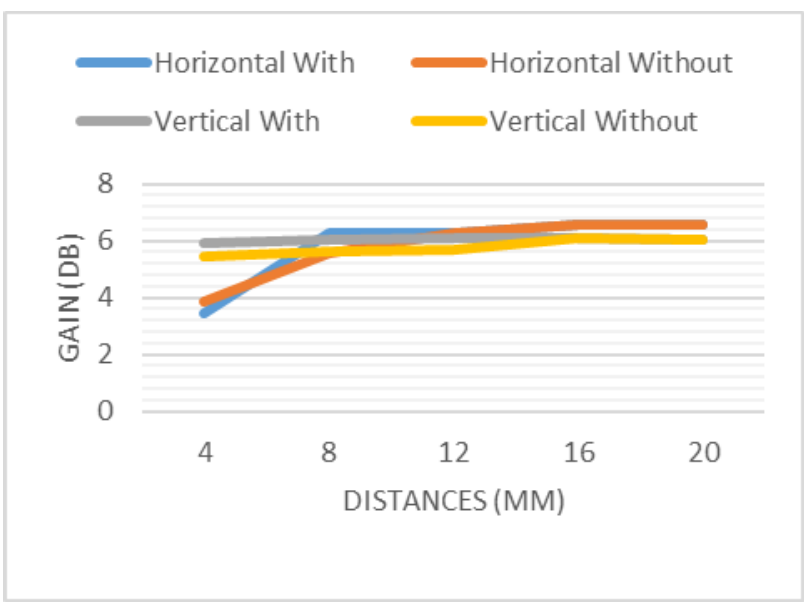

Figure 5: Gain of antenna in horizontal and vertical orientation at $2.45 \mathrm{GHz}$

\section{Conclusion}

The result presented in this paper shows that the presence of metallic loop like accessory affects the SAR and the reflection coefficient of the planar wearable textile monopole. Besides, the antenna orientations and locations produce a prominent effect on SAR value. But,the value of gain also increased when the vertically polarization. SAR is increased when the TM antenna is horizontally orientated than when the antenna is vertically polarized in frequency $2.45 \mathrm{GHz}$.

\section{References}

1. Islam, M. T., Abidin, H. Z., Faruque, M. R. I. \& Misran, N. Analysis of Materials Effects on Radio Fre- Quency Electromagnetic Fields in Human Head. Prog. Electromagn. Res. 128, 121-136 (2012).

2. Safari, M. \& Abdolali, A. Dental Implants and Mobile-Phone Use. 43-51

3. Source, A. E. Comparison on the Effect of Homogeneous and Inhomogeneous Body on Antenna Performance and SAR. 62-64 (2012).

4. Zhu, C., Wu, D., Ping, L. \& Liang, W. The Effects of Head Parameters on the Specific Absorption Rate in the Human Head. 423-427 (2015).

5. Elias, N. A., Samsuri, N. A., Rahim, M. K. A., Othman, N. \& Jalil, M. E. Effects of Human Body and Antenna Orientation on Dipole Textile Antenna Performance and SAR. 132-136 
(2012).

6. Sriprasoet, C. Effects of Ring Metallic Objects on Specific Absorption Rate in Human Head Caused by Mobile Phone. 1-6 (2015).

7. Rahim, H. A., Abdulmalek, M., Soh, P. J. \& Vandenbosch, G. A. E. Evaluation of a broadband textile monopole antenna performance for subject-specific on-body applications. Appl. Phys. A 123, 97 (2017).

8. Ronald, S. H. et al. Designing Asian-Sized Hand Model for SAR Determination at GSM900/1800: Simulation Part. Prog. Electromagn. Res. 129, 439-467 (2012). 